\title{
Patients with schizophrenia show deficits of working memory maintenance components in circuit-specific tasks
}

\author{
David Zilles • Eva Gruber • Peter Falkai • \\ Oliver Gruber
}

Received: 25 July 2009/Accepted: 3 February 2010/Published online: 19 February 2010

(C) The Author(s) 2010. This article is published with open access at Springerlink.com

\begin{abstract}
Working memory (WM) deficits are a neuropsychological core finding in patients with schizophrenia and also supposed to be a potential endophenotype of schizophrenia. Yet, there is a large heterogeneity between different WM tasks which is partly due to the lack of process specificity of the tasks applied. Therefore, we investigated WM functioning in patients with schizophrenia using process- and circuit-specific tasks. Thirty-one patients with schizophrenia and 47 controls were tested with respect to different aspects of verbal and visuospatial working memory using modified Sternberg paradigms in a computer-based behavioural experiment. Total group analysis revealed significant impairment of patients with schizophrenia in each of the tested WM components. Furthermore, we were able to identify subgroups of patients showing different patterns of selective deficits. Patients with schizophrenia exhibit specific and, in part, selective WM deficits with indirect but conclusive evidence of dysfunctions of the underlying neural networks. These deficits are present in tasks requiring only maintenance of verbal or visuospatial information. In contrast to a seemingly global working memory deficit, individual analysis revealed differential patterns of working memory impairments in patients with schizophrenia.
\end{abstract}

Keywords Neurocognitive functioning . Working memory · Schizophrenia

D. Zilles $(\bowtie) \cdot$ E. Gruber · P. Falkai · O. Gruber Department of Psychiatry and Psychotherapy, Georg August University, Von-Siebold-Str. 5, 37075 Goettingen, Germany

e-mail: dzilles@gwdg.de

\section{Introduction}

Schizophrenia is a severe mental disorder that usually occurs in early adulthood with a lifetime prevalence of about $1 \%$. It is characterized by positive (delusions and hallucinations) as well as negative (blunted affect, psychomotor retardation, apathy) symptoms. Another frequent finding is a broad range of cognitive impairments [21], including lack of concentration and attention, problems in memory and learning and executive functions. Especially working memory function, which comprises the maintenance and manipulation of information over a short period of time, has been proven to be a core deficit of the disorder [30]. Since working memory is considered as a basic mental function underlying many other higher cognitive functions such as reasoning, learning and comprehension, it is of particular interest for psychiatric research, especially with respect to schizophrenic psychoses. In contrast to the broad and heterogeneous spectrum of illness phase-dependent symptoms, the neuropsychological impairments seem to remain stable over the course of the disorder [20]. Thus, working memory is suggested to be a promising endophenotype for schizophrenia [11] and as such could represent a link between the clinical symptoms of the disease and genetic or other aetiological factors. Cognitive endophenotypes are thought to be influenced more directly by the genetic basis of the disorder and to be under the influence of a smaller number of genes. Indeed, there is a growing evidence for associations between specific genetic polymorphisms and cognitive dysfunction in schizophrenia [32]. Furthermore, specific single nucleotide polymorphisms are correlated with cerebral activation during working memory task performance [28]. Thus, an exact characterization of specific working memory processes and their disturbances in schizophrenia could prove helpful in the search for 
schizophrenia risk genes. Beyond that there is certain evidence that the investigation of endophenotypes could help to improve differential diagnosis between psychiatric disorders. For example, prior studies were able to show differential working memory impairments in patients with schizophrenia and schizoaffective disorder [14] and a differential influence of familial loading on working memory performance in these two diagnostic groups [34].

Human working memory has been hypothesized to be no unitary system but to consist of different specialized subsystems: the phonological loop for the maintenance of verbal information, the visuospatial sketchpad for the maintenance of spatial information, a central executive serving as a supervisory control system and the so-called episodic buffer as an interface between working memory subsystems and long-term memory [4]. Previous functional MRI studies have indeed identified several dissociable neural networks underlying specific verbal and visuospatial working memory functions in healthy individuals $[12,13$, 16-18]. In one study [18], for instance, the performance of a verbal working memory task requiring an articulatory rehearsal mechanism elicited activation in a left-lateralised network including premotor and parietal brain regions, whereas performance of specific visuospatial working memory tasks activated a bilateral brain system including the cortices along posterior parts of the superior frontal sulci and along the intraparietal sulci.

Based upon these former studies investigating healthy individuals, a further large series of subsequent fMRI studies in clinical samples [19, 22-24] as well as complementary lesion studies [15], clear brain-behaviour relationships could be established between specific brain circuits and subcomponents of working memory in humans.

A recent meta-analysis confirmed pronounced working memory deficits in patients with schizophrenia across all working memory domains and different working memory measures [10] and thus again provided support for the presence of broad working memory deficits in schizophrenia. However, there is still the problem of a poor comparability of the different working memory tasks as the different test paradigms markedly differ with respect to their complexity (maintenance, manipulation or the additional requirement of executive functions) leading to a lack of process specificity. For the further investigation of working memory and its subprocesses, it is thus reasonable to use well-characterized, process-specific neuropsychological tasks that are validated with respect to their neural basis.

In the present study, we used such process-specific tasks probing working memory functioning with respect to maintenance of verbal and visuospatial information, respectively. The experimental design that was here applied to a sample of patients with schizophrenia was the same as in our former fMRI studies in which we were able to characterize the neural networks underlying specific working memory components in healthy subjects [18]. On the basis of the established brain-behaviour relationships as outlined above $[12,13,16-19,22-24]$, we were able to indirectly attribute specific working memory deficits of patients with schizophrenia on the behavioural level to dysfunctions of these underlying neural networks.

Beyond the problems with task specificity, there is a debate on a possible differential working memory impairment in patients with schizophrenia. E.g. several studies have stated a greater impairment in tasks requiring higher executive demands [27] compared to maintenance-only tasks with some authors even reporting a lack of significant differences between patients with schizophrenia and healthy controls in the latter [8, 29].

The aim of the present study was to investigate patients with schizophrenia with respect to their performance in process- and circuit-specific verbal and visuospatial working memory maintenance tasks. In contrast to the previous fMRI studies, we now tested the functional integrity of working memory processes in terms of possible behavioural deficits in a larger sample of patients with schizophrenia. Our hypothesis was that patients with schizophrenia would perform worse in verbal as well as visuo-spatial working memory maintenance tasks in comparison with healthy controls.

Second, we sought to identify subgroups of patients possibly showing differential patterns of specific working memory deficits.

Third, we investigated possible correlations between test performance and clinical variables and psychopathology.

\section{Materials and methods}

\section{Subjects}

Thirty-one patients and 47 healthy control subjects were included in this study. The patient group was recruited from inpatients with diagnosis of schizophrenia according to ICD-10 criteria, hospitalized in the Psychiatric Department of the Saarland University Hospital. Healthy comparison subjects were recruited from friends and relatives of the investigators, so we were able to rule out relevant psychiatric disorders without an extensive screening procedure.

Inclusion criteria for patients were a diagnosis of schizophrenia and age from 18 to 65 years. Exclusion criteria were acute suicidality, involuntary treatment, current substance abuse, history of brain trauma, diseases with alterations of cerebral metabolism, uncorrected visual or 
auditory disability and mental retardation. Criteria for healthy comparison subjects were the same as above plus the absence of any past or present psychiatric disorder. All subjects gave written informed consent before participation, and the study was approved by the local ethics committee.

All subjects underwent a computer-based behavioural experiment described below. The patients' clinical state was assessed on the day of the experiment by using Clinical Global Impression Scale (CGI), Beck Depressions Inventory (BDI), Montgomery Asberg Depression Scale (MADRS), Positive and Negative Syndrome Scale (PANSS). On all scales, higher scores reflect greater severity of symptoms.

After a careful matching with respect to age, gender and years of education, statistical analyses were performed including 26 patients and 26 healthy controls. All 26 patients were under antipsychotic medication. Twentythree received second generation antipsychotics (risperidone, olanzapine, amisulpride, clozapine, aripiprazole, ziprasidone), three received first generation antipsychotics (haloperidole, flupentixole). Nine patients were additionally treated with benzodiazepines (low dose lorazepame), three were treated with biperidene. Illness duration ranged from 0 to 24 years (mean 8.09 years, standard deviation 6.99 years), six patients experienced their first psychotic episode. For symptom severity, see Table 1.

\section{Experimental design}

Testing was conducted in an experimental neuropsychological laboratory under standardized conditions. The behavioural experiment consisted of four tasks, testing working memory with respect to verbal and visuospatial components, each under single task conditions (i.e. active rehearsal of the sample items) as well as under dual-task conditions (i.e. under articulatory or visuospatial suppression). These different task conditions were conducted in a blocked manner, i.e. each of these specific tasks was repeated 50 times with a short pause after 25 trials. The order of the tasks performed was counterbalanced across subjects.

In the 'verbal rehearsal task', subjects were instructed to vocalize four sample letters internally one time in the presentation period and then to intensely rehearse them throughout the delay period. In the 'non-articulatory phonological maintenance task', subjects were again instructed to vocalize the four sample letters one time but then to silently count "one, two, three, four, one, two..." and so on during the delay period. This articulatory suppression task has been proven to selectively interfere with verbal working memory $[3,18]$. Subjects were explicitly instructed not to use any visual memory strategies but to concentrate on
Table 1 Group differences between patients with schizophrenia $(n=26)$ and healthy control subjects $(n=26)$ with respect to demographic factors and to performance in different working memory tasks

\begin{tabular}{|c|c|c|c|c|c|}
\hline \multirow{2}{*}{$\begin{array}{l}\text { Working memory task } \\
\text { performance (percentage } \\
\text { correct) }\end{array}$} & \multicolumn{2}{|c|}{$\begin{array}{l}\text { Comparison } \\
\text { subjects }\end{array}$} & \multicolumn{2}{|c|}{ Patients } & \multirow[t]{2}{*}{$P$} \\
\hline & Mean & SD & Mean & SD & \\
\hline Verbal rehearsal & 92.4 & 5.7 & 84.0 & 10.4 & 0.001 \\
\hline $\begin{array}{l}\text { Non-articulatory maintenance of } \\
\text { phonological information }\end{array}$ & 85.4 & 9.1 & 78.6 & 9.7 & 0.012 \\
\hline Visuospatial rehearsal & 90.1 & 6.0 & 80.1 & 13.4 & 0.001 \\
\hline Visuospatial pattern maintenance & 87.6 & 8.5 & 78.1 & 10.7 & 0.001 \\
\hline \multicolumn{6}{|l|}{ Demographic factors } \\
\hline Age (years) & 39.2 & 12.8 & 37.6 & 10.7 & 0.616 \\
\hline Gender (M/F) & $16 / 10$ & & $16 / 10$ & & 1.000 \\
\hline Years of education & 13.4 & 2.7 & 12.8 & 2.8 & 0.425 \\
\hline \multicolumn{6}{|l|}{ Symptom severity } \\
\hline CGI & & & 4.04 & 1.08 & \\
\hline MADRS & & & 12.00 & 8.35 & \\
\hline PANSS-P & & & 10.92 & 4.77 & \\
\hline PANSS-N & & & 13.15 & 4.32 & \\
\hline
\end{tabular}

$S D$ standard deviation; $M$ male; $F$ female

The bold-faced $P$ values are significant at level $P<0.05$

the mere sound of the letters. In addition, letter case was systematically changed between presentation and response phase to prevent the subjects from using a visual strategy. In the 'visuospatial rehearsal task', subjects got the instruction to repetitively perform overt shifts of attention to the four spatial positions in a $5 \times 5$ matrix that they had to remember. This working memory strategy has been termed a 'visuospatial rehearsal mechanism' by some authors [2]. Finally, in the 'visuospatial pattern maintenance task', subjects were instructed to remember the spatial pattern built by the four sample positions and then perform a visuospatial suppression task during the delay period by following a little moving star with their eyes. This procedure had been proven to selectively interfere with visuospatial working memory $[3,18]$. For each task, there was a practice phase to ensure that participants had understood task instructions.

Stimulus presentation was identical for each of the four task conditions. At the beginning of each trial, a $5 \times 5$ matrix appeared on the monitor with four squares of the matrix filled by different letters, randomly chosen out of a set of eight, in German, phonologically similar letters (B, C, D, E, G, P, T, W). The position of the letters in the matrix was also chosen randomly, with the constraint that no adjacent squares should be filled by letters, to avoid the encoding of two or more squares as a single item. This presentation was visible on the screen for $2 \mathrm{~s}$ followed by a 
delay period of $3.2 \mathrm{~s}$, in which only the empty matrix could be seen on the monitor together with a little star quickly moving across the screen. Simultaneously, rhythmic $4-\mathrm{kHz}$ tones were presented throughout the delay period with a repetition frequency of $3.3 \mathrm{~Hz}$ to set the pace of the rehearsal or counting. During this interval, subjects had to keep the letters or the spatial positions in mind using the above described memory strategies. In the following response phase, one letter was presented in a square of the matrix for $2 \mathrm{~s}$. Subjects had to decide whether the probe letter (verbal working memory task) or its position (visuospatial working memory task) matched one of the four target letters or positions, respectively, presented at the beginning. There was an additional 2-s intertrial interval between the single trials during which a fixation cross was presented on the screen.

\section{Statistical analysis}

Statistical analyses were performed using SPSS for Windows (version 11.0.1). According to our a priori hypothesis, inter-group comparisons were performed using independent, unpaired, two-tailed $t$-tests using a statistical threshold of $P<0.05$ for each of the four task conditions. Possible relationships between task performance and clinical variables were tested with Pearson correlations, with a significance level of $P<0.05$, corrected for multiple comparisons.

\section{Results}

\section{Working memory performance}

Both healthy and schizophrenic subjects performed better in the single task (i.e. rehearsal) conditions when compared to the tasks requiring verbal or visuospatial suppression. Statistical significance was reached in the verbal tasks (patients: $P=0.001$, controls: $P<0.0005)$. Standard deviations in most of the tasks were remarkably larger in the patient group.

Whole group analysis revealed significant differences in the percentage of correct answers between the schizophrenia $(n=31)$ and the comparison group $(n=47)$ with respect to all tested working memory components. However, these groups differed significantly in their educational level (years of education), which was proven to be correlated with working memory performance in a larger sample of healthy controls $(n=59$; articulatory rehearsal: $r=0.37, P=0.004$; visuo-spatial rehearsal: $r=0.42$, $P=0.001)$. Nevertheless, the differences in working memory performance remained significant when groups were matched with respect to gender, age and years of education (26 patients vs. 26 healthy comparison subjects, see Table 1).

\section{Subgroup analysis}

Total group analysis thus showed a general impairment of working memory performance for the schizophrenia group. However, as there was a high degree of interindividual heterogeneity within the patient group, we aimed to identify subgroups of patients showing different patterns of selective deficits. As a definition for these individual deficits, we chose a deviation of minus one standard deviation from the mean of the percentage of correct answers in the comparison group. Using this criterion, 23 out of 26 subjects showed test deficits. In four subjects, we observed an isolated deficit in the verbal rehearsal task while they showed a numerically even better performance compared to the healthy control group in the visuospatial tasks. Six other patients showed a selective deficit in visuospatial working memory while performing numerically better than the controls in the verbal working memory tasks. Despite the small sample size in the comparison of these two subgroups, we confirmed significant differences with respect to their verbal and visuospatial working memory test performance, respectively (see Fig. 1).

Associations between working memory performance and clinical variables

Correlation analyses were conducted to examine associations between patients' task performance, age, gender, years of education, duration of illness and hospitalization and severity of symptoms according to the psychopathological scales mentioned above. After correction for multiple testing, we did not find any significant correlations between performance rates in the different working memory tasks and these demographic and clinical variables.

\section{Discussion}

There is an extensive literature reporting impaired working memory function in patients with schizophrenia [e.g. 9, 26, 29, 33]. A problem is the large variety of behavioural experiments used to assess working memory and the lack of process specificity, hindering the comparability of the results of these studies and the possibility to consistently identify underlying neural networks. While previous fMRI studies using n-back tasks could not reveal domain-specific patterns of brain activation related to verbal and visuospatial working memory $[7,35]$, other studies using more appropriate item-recognition tasks were able to demonstrate the dissociability of brain systems that underlie these 


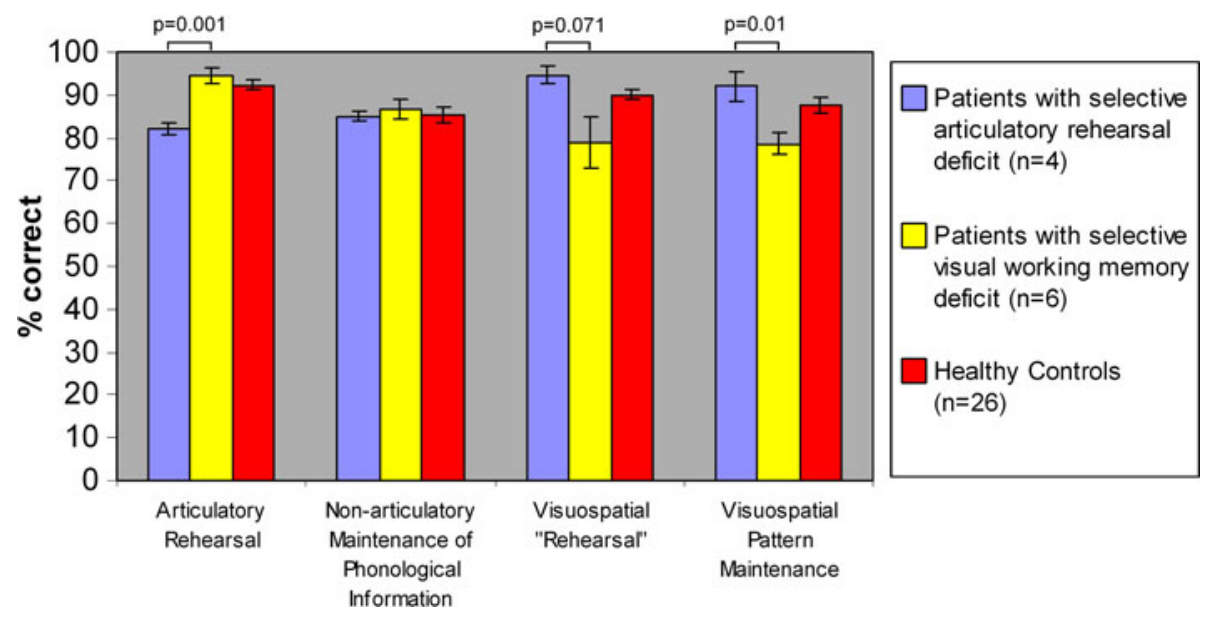

Fig. 1 Differential working memory deficits in subgroups of patients with schizophrenia. Comparison between the two schizophrenia subgroups built by deficit patterns revealed significant differences in working memory performance. Both patient groups depicted here show a selective deficit in only one working memory domain while

different components of working memory [18, 31]. Consequently, in the present study, we used the same experimental design as in one of these latter studies [18] in order to be able to attribute deficits in working memory performance to previously identified neural networks.

The present study confirmed the findings of earlier investigations in patients with schizophrenia showing a marked impairment of both verbal and visuospatial working memory functioning. According to our results, this deficit is also present in maintenance-only tasks. To date, there is an ongoing debate on the presence and extent of working memory impairment in patients with schizophrenia in tasks requiring maintenance only [5]. For example, an early study on working memory performance in schizophrenia did not detect a deficit in the digit span task [29], just as a more recent study [8]. Beyond that, some other authors reported greater impairment in more demanding manipulation tasks [25, 27]. These latter studies, however, also revealed a worse performance in maintenance-only tasks. With respect to unaffected relatives of patients with schizophrenia, Conklin [6] reported an impaired performance in tasks with higher executive processing requirements. Nevertheless, in the same study, patients showed impairments in all tasks irrespective of processing demands, including both the verbal and the visuospatial domain tested by digit and spatial span. Moreover, this evidence of a deficit in the working memory maintenance component is supported by two meta-analyses $[1,10]$ showing moderate to large effect sizes in tasks without any manipulation or 'executive' requirements. According to the study by Aleman [1], there was no significant difference between the impairment in the digit span forward and backward. Thus, though there is some their performance in the other domain is unimpaired. The figure shows the means and standard errors of the two patient groups and the healthy control group. The indicated $P$-values arise from a two-group comparison of the two patient subgroups

evidence for an especially pronounced impairment in working memory tasks with higher demands, maintenanceonly processes also seem to be affected and our current findings provide further support for a deficit in both verbal and visuospatial working memory maintenance in groups of patients with schizophrenia.

Furthermore, our data suggest that these deficits in terms of significant statistical effects on the group level are not necessarily the result of a general working memory dysfunction in the individual patient. Overall group analysis revealed deficits with respect to all tested working memory components (Table 1), which could be interpreted as a global working memory deficit or even a more general cognitive impairment. However, in addition to this seemingly global deficit, we found a high degree of interindividual variability of test performance. In a subsequent and more detailed analysis, we searched for subgroups of patients possibly showing more specific deficits. Indeed, we were able to identify such subgroups with some patients exhibiting differential patterns of working memory deficits (e.g. an isolated impairment in the verbal rehearsal task). Thus, our data provide evidence for a high interindividual variability with regard to the presence or absence of specific working memory maintenance deficits in patients with schizophrenia and for the existence of patient subgroups with isolated deficits in only one domain of working memory.

This finding of a high interindividual variability in working memory functioning may also reflect the presumed heterogeneity of aetiopathogenesis and pathophysiology for schizophrenic disorders. Furthermore, heterogeneity on the individual level may also contribute to the heterogeneity that was found on the group level in a 
current metaanalysis [10]. Future studies should therefore aim to study more homogeneous subgroups of patients with schizophrenia. Our current approach of identifying differential impairments of working memory functioning via experimental neuropsychological testing provides a possible method for the generation of endophenotypically defined subgroups of psychiatric patients. Groups that are built on this basis could be more homogeneous not only with respect to the pattern of cognitive deficits but also with respect to their underlying aetiology and pathophysiology.

Taking into account our previous fMRI findings in healthy individuals $[12,13,17,18]$ and psychiatric patients $[19,22-24]$ and the fact that there are subgroups of patients presenting isolated deficits in only one working memory domain, we infer that specific dysfunctions of the different underlying neuronal networks contribute to the performance deficit on the behavioural level. Of course, our present study can only provide indirect evidence for this, but the validity of this conjecture is supported by lesion studies as well. Two patients with focal brain damages, one with an isolated lesion to Broca's area and the other with a selective bilateral lesion to anterior parts of the middle frontal gyrus, showed a double dissociation of specific working memory deficits. The former was impaired in a task requiring verbal rehearsal while performing normal during non-articulatory maintenance of verbal information, whereas the latter showed the opposite pattern of impairment [15]. Taken together, because brain-behaviour relationships had been carefully established by converging evidence from numerous functional neuroimaging and lesion studies, this allowed us to infer from specific impairments in working memory subfunctions the underlying dysfunctions of specific brain regions or networks.

Important to note, the subgroups that were determined according to these specific patterns of working memory deficits did not differ with respect to any clinical characteristics in our sample. Also, in the total group analysis, there were no significant correlations between test performance and clinical variables and psychopathology scores. This finding is consistent with the notion that working memory deficits are considered to be potential endophenotypes, i.e. trait markers that are independent of fluctuations in the clinical status of the disorder.

Of course our findings are limited by the relative small sample size. Thus, the validity of the identified working memory deficit patterns should be confirmed in larger samples. The same applies to the retest-reliability of the observed deficits. Furthermore, we cannot rule out medication effects on working memory performance. To further investigate a potential and maybe differential effect of different antipsychotics on the different working memory subsystems, a larger sample size would also be needed.
Finally, further investigation is needed to identify neurobiological (e.g. genetic) markers in the patient subgroups that could provide evidence for a distinct aetiology and pathophysiology.

In conclusion, the results of the present study strengthen the evidence for an impairment of the maintenance component of both verbal and visuospatial working memory in patients with schizophrenia. Moreover, our data provide evidence for a high degree of interindividual variability and for the existence of subgroups of patients with schizophrenia showing differential working memory deficits.

Open Access This article is distributed under the terms of the Creative Commons Attribution Noncommercial License which permits any noncommercial use, distribution, and reproduction in any medium, provided the original author(s) and source are credited.

\section{References}

1. Aleman A, Hijman R, de Haan EHF, Kahn RS (1999) Memory impairment in schizophrenia: a meta-analysis. Am J Psychiatry 156:1358-1366

2. Awh E, Jonides J, Reuter-Lorenz PA (1998) Rehearsal in spatial working memory. J Exp Psychol Hum Percept Perform 24:780 790

3. Baddeley AD (1986) Working memory. Oxford University Press, Oxford, UK

4. Baddeley A (2003) Working memory: looking back and looking forward. Nat Rev Neurosci 4:829-839

5. Barch DM, Smith E (2008) The cognitive neuroscience of working memory: relevance to CNTRICS and schizophrenia. Biol Psychiatry 64:11-17

6. Conklin HA, Curtis CE, Calkins ME, Iacono WG (2005) Working memory functioning in schizophrenia patients and their firstdegree relatives: cognitive functioning shedding light on etiology. Neuropsychologia 43:930-942

7. D'Esposito M, Aguirre GK, Zarahn E, Ballard D, Shin RK, Lease J (1998) Functional MRI studies of spatial and nonspatial working memory. Cogn Brain Res 7:1-13

8. Fitzgerald D, Lucas S, Redoblado MA, Winter V, Brennan J, Anderson J, Harris A (2004) Cognitive functioning in young people with first episode psychosis: relationship to diagnosis and clinical characteristics. Aust N Z J Psychiatry 38:501-510

9. Fleming K, Goldberg TE, Gold JM, Weinberger DR (1995) Verbal working-memory dysfunction in schizophrenia-use of a Brown-Peterson paradigm. Psychiatry Res 56:155-161

10. Forbes NF, Carrick LA, McIntosh AM, Lawrie SM (2009) Working memory in schizophrenia: a meta-analysis. Psychol Med 39:889-905

11. Gottesman II, Gould TD (2003) The endophenotype concept in psychiatry: etymology and strategic intentions. Am J Psychiatry 160:636-645

12. Gruber O (2001) Effects of domain-specific interference on brain activation associated with verbal working memory task performance. Cereb Cortex 11:1047-1055

13. Gruber O, Goschke T (2004) Executive control emerging from dynamic interactions between brain systems mediating language, working memory and attentional processes. Acta Psychol 115:105-121

14. Gruber O, Gruber E, Falkai P (2006) Articulatory rehearsal in verbal working memory: a possible neurocognitive 
endophenotype that differentiates between schizophrenia and schizoaffective disorder. Neurosci Lett 405:24-28

15. Gruber O, Gruber E, Falkai P (2005) Neural correlates of working memory deficits in schizophrenic patients. Ways to establish neurocognitive endophenotypes of psychiatric disorders. Radiologe 45:153-160

16. Gruber O, Muller T, Falkai P (2007) Dynamic interactions between neural systems underlying different components of verbal working memory. J Neural Transm 114:1047-1050

17. Gruber O, von Cramon DY (2001) Domain-specific distribution of working memory processes along human prefrontal and parietal cortices: a functional magnetic resonance imaging study. Neurosci Lett 297:29-32

18. Gruber O, von Cramon DY (2003) The functional neuroanatomy of human working memory revisited-evidence from 3-T fMR1 studies using classical domain-specific interference tasks. Neuroimage 19:797-809

19. Gruber O, Tost H, Henseler I, Schmael C, Scherk H, Ende G, Ruf M, Falkai P, Rietschel M (2009) Pathological amygdala activation during working memory performance: Evidence for a pathophysiological trait marker in bipolar affective disorder. Hum Brain Mapp. 2009 Jul 14 (Epub ahead of print)

20. Heaton RK, Gladsjo JA, Palmer BW, Kuck J, Marcotte TD, Jeste DV (2001) Stability and course of neuropsychological deficits in schizophrenia. Arch Gen Psychiatry 58:24-32

21. Heinrichs RW, Zakzanis KK (1998) Neurocognitive deficit in schizophrenia: a quantitative review of the evidence. Neuropsychology 12:426-445

22. Henseler I, Falkai P, Gruber O (2009) A systematic fMRI investigation of the brain systems subserving different working memory components in schizophrenia. Eur J Neurosci 30:693702

23. Henseler I, Gruber O, Kraft S, Krick C, Reith W, Falkai P (2008) Compensatory hyperactivations as markers of latent working memory dysfunctions in patients with obsessive-compulsive disorder: an fMRI study. J Psychiatry Neurosci 33:209-215

24. Henseler I, Falkai P, Gruber O (2009) Disturbed functional connectivity within brain networks subserving domain-specific subcomponents of working memory in schizophrenia: Relation to performance and clinical symptoms. J Psychiatr Res. 2009 Oct 16 (Epub ahead of print)

25. Horan WP, Braff DL, Nuechterlein KH, Sugar CA, Cadenhead KS, Calkins ME, Dobie DJ, Freedman R, Greenwood TA, Gur RE, Gur RC, Light GA, Mintz J, Olincy A, Radant AD, Schork NJ, Seidman LJ, Siever LJ, Silverman JM, Stone WS, Swerdlow
NR, Tsuang DW, Tsuang MT, Turetsky BI, Green MF (2008) Verbal working memory impairments in individuals with schizophrenia and their first-degree relatives: findings from the consortium on the genetics of schizophrenia. Schizophr Res 103:218-228

26. Keefe RSE, LeesRoitman SE, Dupre RL (1997) Performance of patients with schizophrenia on a pen and paper visuospatial working memory task with short delay. Schizophr Res 26:9-14

27. Kim JH, Glahn DC, Nuechterlein KH, Cannon TD (2004) Maintenance and manipulation of information in schizophrenia: further evidence for impairment in the central executive component of working memory. Schizophr Res 68:173-187

28. Kircher T, Thienel R, Wagner M, Reske M, Habel U, Kellermann T, Frommann I, Schwab S, Wolwer W, von Wilmsdorf M, Braus D, Schmitt A, Rapp A, Stocker T, Shah N, Henn F, Sauer H, Gaebel W, Maier W, Schneider F (2009) Neuregulin 1 ICE-single nucleotide polymorphism in first episode schizophrenia correlates with cerebral activation in fronto-temporal areas. Eur Arch Psychiatry Clin Neurosci 259:72-79

29. Park S, Holzman PS (1992) Schizophrenics show spatial working memory deficits. Arch Gen Psychiatry 49:975-982

30. Silver H, Feldman P, Bilker W, Gur RC (2003) Working memory deficit as a core neuropsychological dysfunction in schizophrenia. Am J Psychiatry 160:1809-1816

31. Smith EE, Jonides J, Koeppe RA (1996) Dissociating verbal and spatial working memory using PET. Cereb Cortex 6:11-20

32. Spellmann I, Muller N, Musil R, Zill P, Douhet A, Dehning S, Cerovecki A, Bondy B, Moller HJ, Riedel M (2008) Associations of SNAP-25 polymorphisms with cognitive dysfunctions in Caucasian patients with schizophrenia during a brief trail of treatment with atypical antipsychotics. Eur Arch Psychiatry Clin Neurosci 258:335-344

33. Wexler BE, Stevens AA, Bowers AA, Sernyak MJ, GoldmanRakic PS (1998) Word and tone working memory deficits in schizophrenia. Arch Gen Psychiatry 55:1093-1096

34. Zilles D, Burke S, Schneider-Axmann T, Falkai P, Gruber O (2009) Diagnosis-specific effect of familial loading on verbal working memory in schizophrenia. Eur Arch Psychiatry Clin Neurosci 259:309-315

35. Zurowski B, Gostomzyk J, Gron G, Weller R, Schirrmeister H, Neumeier B, Spitzer M, Reske SN, Walter H (2002) Dissociating a common working memory network from different neural substrates of phonological and spatial stimulus processing. Neuroimage 15:45-57 